\title{
Applying Compressive Sensing to the Massive MIMO Channel Estimation Problem
}

\author{
Felipe Augusto Pereira de Figueiredo \\ Ghent University - imec, IDLab, Department of Information Technology, Ghent, Belgium. \\ Email: felipe.pereira@ugent.be
}

\begin{abstract}
This paper proposes the use of compressive sensing to tackle the Massive MIMO channel estimation problem. As our results show compressive sensing-based estimators perform as well as the optimum MMSE estimator.

Keywords: massive MIMO; compressive sensing; channel estimation

\section{INTRODUCTION}

Accurate channel estimation is very important for massive MIMO systems, once they are necessary to provide significant improvements in spectral and energy efficiency. In massive MIMO systems, the base station (BS) estimates the channels of all its connected users. These estimates, whcih are obtained during the uplink transmission phase are used to generate pre-coding and decoding matrices. These matrices are used to receive and transmit data. Therefore, accurate estimation of the channels is a very important task for massive MIMO systems. In this work, we present a comparative study on the application of compressive sensing to the massive MIMO channel estimation problem.
\end{abstract}

\section{SySTEM Model}

Let $x_{k}(n)$ denote the transmitted time-domain samples of the $k$ th MTC device, $k=1, \ldots, K$, i.e. the Orthogonal Frequency-Division Multiplexing (OFDM) symbol transmitted by the $k$ th MTC device. OFDM symbols are normalized to unitary variance, so $E\left[\left|x_{k}(n)\right|^{2}\right]=1$. In the uplink, the signals from a cluster of $K$ MTC devices is collected into the vector

$$
\mathbf{x}(n)=\left[x_{1}(n), \ldots, x_{K}(n)\right]^{T},
$$

where $(\cdot)^{T}$ denotes transposition and $\mathbf{x} \in \mathbb{C}^{K \times 1}[16]$.

Consider now a Massive MU-MIMO setup, where $\mathbf{x}(n)$ is detected by a BS equipped with $M$ receive antennas, $M \gg K$. Between every transmit antenna $k$ at the MTC device and every receive antenna $m$ at the BS there is a complex singleinput single-output (SISO) channel impulse response $h_{m, k}(n)$ of length $L+1$, described by the vector:

$$
\mathbf{h}_{m, k}=\left[h_{m, k}(0), \ldots, h_{m, k}(L)\right]^{T} .
$$

Under the assumption that all SISO channels have channel or$\operatorname{der} L$, the frequency selective MIMO channel can be described by a number of $L+1 M \times K$ complex channel matrices:

$$
\mathbf{H}(n)=\left[\begin{array}{ccc}
h_{1,1}(n) & \cdots & h_{1, K}(n) \\
\vdots & \ddots & \vdots \\
h_{M, 1}(n) & \cdots & h_{M, K}(n)
\end{array}\right], n=0, \ldots, L
$$

If the signal received by the $m$ th antenna at the $n$th time instant is denoted by $y_{m}(k)$, the signals received by all $M$ antennas can be represented in vector form as

$$
\mathbf{y}(n)=\left[y_{1}(n), \ldots, y_{M}(n)\right]^{T}
$$

which we then rewrite in terms of (1) and (3) as

$$
\mathbf{y}(n)=\sum_{i=0}^{L} \mathbf{H}(i) \mathbf{x}(n-i)+\mathbf{w}(n),
$$

where the noise vector $\mathbf{w}(n)$ has length $M$ is assumed additive white Gaussian noise (AWGN) with zero mean and variance $\sigma_{w}^{2}$ per receive antenna. For each of the $K$ MTC devices there are $M$ PNSCH signal versions in (5). Hence, the task of the BS consists of detecting $K$ simultaneous MTC transmissions on the basis of estimates of the channel coefficients in (3).

\section{Channel Estimation}

The Massive MIMO channel is modeled as a superposition of $M \times K$ single-input single-output (SISO) channels. Each SISO channel has $L+1$ unknowns. For pilot-assisted channel estimation in OFDM systems we will employ the comb-type pilot pattern on the time-frequency 2-D grids [17].

Consider an OFDM system with $N$ subcarriers in each OFDM symbol, among which $N_{p}$ pilot subcarriers indicated by $p_{1}, p_{2}, \ldots, p_{N_{p}}$ are used for frequency-domain pilotassisted channel estimation. Without loss of generality, we assume that $1 \leq p_{1}<p_{2}<\ldots<p_{N_{p}}<N$. The corresponding transmit pilot symbols are denoted as $s\left(p_{1}\right), s\left(p_{2}\right), \ldots, s\left(p_{N_{p}}\right)$. Let $h(0), h(1), \ldots, h(L+1)$ be the equivalent discrete channel impulse response (CIR) with the maximum multipath delay spread being $L+1$ samples. The received signals on the pilot subcarriers can be written as

$$
\begin{aligned}
{\left[\begin{array}{c}
y\left(p_{1}\right) \\
y\left(p_{2}\right) \\
\vdots \\
y\left(p N_{p}\right)
\end{array}\right]=} & {\left[\begin{array}{cccc}
s\left(p_{1}\right) & 0 & 0 & 0 \\
0 & s\left(p_{2}\right) & 0 & 0 \\
0 & 0 & \ddots & 0 \\
0 & 0 & 0 & s\left(p_{N_{p}}\right)
\end{array}\right] } \\
& . \mathbf{F}_{N_{p} \times L+1}\left[\begin{array}{c}
h(0) \\
h(1) \\
\vdots \\
h(L+1)
\end{array}\right]+\left[\begin{array}{c}
w(1) \\
w(2) \\
\vdots \\
w\left(N_{p}\right)
\end{array}\right]
\end{aligned}
$$

where $w(i) \sim \mathcal{C N}\left(0, \sigma_{w}^{2}\right), i=1,2, \ldots, N_{p}$ is the i.i.d additive white Gaussian noise, and $\mathbf{F}_{N_{p} \times L+1}$ is a discrete Fourier 
transform (DFT) sub-matrix given by

$$
\mathbf{F}_{N_{p} \times L+1}=\frac{1}{\sqrt{N}}\left[\begin{array}{cccc}
1 & w^{p_{1}} & \cdots & w^{p_{1}(L-1)} \\
1 & w^{p_{2}} & \cdots & w^{p_{2}(L-1)} \\
\vdots & \vdots & \ddots & \vdots \\
1 & w^{p_{N_{p}}} & \cdots & w^{p_{N_{p}}(L-1)}
\end{array}\right]
$$

where $w=e^{-j 2 \pi / N}$. We denote

$$
\begin{aligned}
& \mathbf{S} \triangleq \operatorname{diag}\left\{s\left(p_{1}\right), s\left(p_{2}\right), \ldots, s\left(p_{N_{p}}\right)\right\} \\
& \mathbf{y} \triangleq\left[y\left(p_{1}\right), y\left(p_{2}\right), \ldots, y\left(p_{N_{p}}\right)\right]^{T} \\
& \mathbf{h} \triangleq[h(0), h(1), \ldots, h(L+1)]^{T} \\
& \mathbf{w} \triangleq\left[w(1), w(2), \ldots, w\left(N_{p}\right)\right]^{T} .
\end{aligned}
$$

Furthermore, we let

$$
\mathbf{D} \triangleq \mathbf{S F}_{N_{p} \times L+1} .
$$

Then, (6) can be rewritten as

$$
\mathbf{y}=\mathbf{D h}+\mathbf{w} .
$$

Next we briefly describe the channel estimation techniques adopted here for comparison. They are employed to estimate one of the SISO channels.

\section{A. Least Squares}

The linear Least Square (LS) channel estimator is given by

$$
\hat{\mathbf{h}}_{\mathbf{m}, \mathbf{k}}^{\mathbf{L S}}=\left[\mathbf{D}^{\dagger} \mathbf{D}\right]^{-1} \mathbf{D}^{\dagger} \mathbf{y}_{m}
$$

where $(.)^{\dagger}$ denotes transpose-conjugate (Hermitian) operation.

LS employs no knowledge of the statistics of the channels. It presents very low complexity, but has a high mean-square error [17].

\section{B. Minimum Mean Squared Error}

$$
\hat{\mathbf{h}}_{\mathbf{m}, \mathbf{k}}^{\mathrm{MMSE}}=\left[\mathbf{D}^{\dagger} \mathbf{D}+\frac{\sigma_{n}^{2}}{\sigma_{h}^{2}} \mathbf{I}\right]^{-1} \mathbf{D}^{\dagger} \mathbf{y}_{m}
$$

where $\sigma_{n}^{2}$ is the noise variance and $\sigma_{h}^{2}$ is the variance of the SISO channel, $\mathbf{h}_{m, k}$. MMSE estimators employ second-order statistics of the channels in order to minimize the mean-square error. These estimators present better performance than the LS ones, especially at low SNR values [17].

\section{Compressed Sensing}

The technique for sparse signal recover known as compressive sensing has been under heavy investigation since its inception a few years ago $[18,19]$. Sparse channel estimation can be more efficient than the conventional channel estimation approaches, i.e., LS, MMSE, etc., due to the sparse nature of multipath wireless channels [20,21].

\section{Signal Detection}

Detection techniques are needed to separate the data streams transmitted by each MTC device in our Massive MU-MIMO setup. Maximum likelihood detection is theoretically optimum but its complexity grows exponentially with the modulation order and the number of transmit antennas $K$ (hard to implement in case of thousands of MTC devices). One way to circumvent this limitation is to use sub-optimal alternatives with reduced computational complexity [22]. Maximum Ratio Combining (MRC) chooses the linear detection matrix using $\mathbf{A}_{\mathrm{MRC}}=\mathbf{H}$, which requires $\mathcal{O}(M K)$ multiplications. Constrained to $\mathbf{A H}=$ I, Zero Forcing (ZF) chooses $\mathbf{A}_{\mathrm{ZF}}=\mathbf{H}\left(\mathbf{H}^{\dagger} \mathbf{H}\right)^{-1}$ and poses an associated complexity of $\mathcal{O}\left(M K+M K^{2}+K^{3}\right)$ [23].

In contrast to ZF, which minimizes interference but fails to treat noise, and to MRC, which minimizes noise but fails to treat interference, MMSE achieves an optimal balance between interference suppression and noise enhancement at the same cost of ZF [23, 24]. As the name suggests, the MMSE detector chooses the $\mathbf{A}$ that minimizes $e=E\left[\left\|\mathbf{A}^{\dagger} \mathbf{y}-\mathbf{x}\right\|^{2}\right]$ without any additional constraints

$$
\mathbf{A}_{\mathrm{MMSE}}=\mathbf{H}\left(\mathbf{H}^{\dagger} \mathbf{H}+\frac{\sigma_{n}^{2}}{\sigma_{x}^{2}} \mathbf{I}\right)^{-1}
$$

where $\sigma_{x}^{2}$ and $\sigma_{n}^{2}$ denote the variances of transmitted signal vector and noise vector, respectively.

\section{Simulation WORK}

In this section, we assess the performances of LS, MMSE and OMP channel estimators in terms of their Mean Square Error (MSE) and Bit Error Rate (BER) over a range of Signalto-Noise Ratios (SNRs).

As can be seen in Figure 1 (a) OMP has a better MSE performance than MMSE and LS channel estimator for the whole range of SNR values. On Figure 1 (b), we see the results of the BER comparison. As we notice, for low SNR values the MMSE estimator performs better than the OMP, however, as the SNR increases, the OMP estimator surpasses the MMSE estimator and for SNR values greater than $30 \mathrm{~dB}$ they both achieve a floor value and from that point on both of them present the same performance. The floor achieve by all the three estimators is caused by the type of combining adopted in this work and it can be mitigated by increasing the number of antennas deployed at the BS.

\section{CONCLUSIONS}

This paper has proposed the use of compressive sensing to tackle the Massive MIMO channel estimation problem. As can be seen by analyzing the results the OMP based estimator performs as well as the MMSE estimator.

\section{REFERENCES}

[1] 4G Americas. 4G Americas' Recommendations on 5G Requirements and Solutions. Online. [Available]: http://www.4gamericas.org/en/ resources/white-papers/

[2] G. Fettweis and S. Alamouti, "5G: Personal Mobile Internet beyond What Cellular Did to Telephony”, IEEE Commun. Mag., vol. 52, no. 2, pp. 140-145, Feb. 2014. 

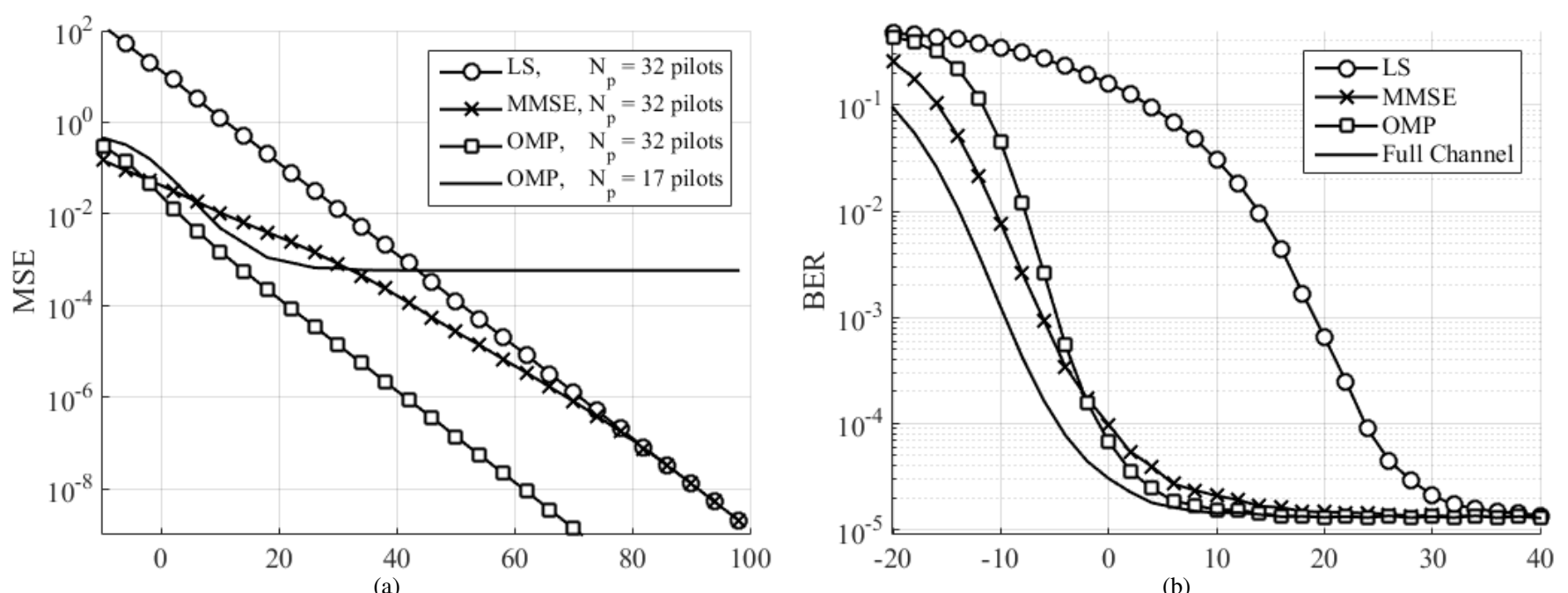

Fig. 1. Comparison of MSE and BER performance for different channel estimation techniques.

[3] G. Wunder et al., "5GNOW: Non-Orthogonal Asynchronous Waveforms for Future Mobile Applications", IEEE Commun. Mag., vol. 52, no. 2, pp. 97-105, Feb. 2014.

[4] A. Elmangoush, H. Coskun, S. Wahle, N. Blum and T. Magedanz, "Promoting M2M Application Development for Smart City, I29th Meeting of the Wireless World Research Forum (WWRF), Oct. 2012.

[5] J. M. Corchado, J. Bajo, D. I. Tapia, and A. Abraham, "Using heterogeneous wireless sensor networks in a telemonitoring system for healthcare, IEEE Trans. on Inf. Technol. Biomed., vol. 14, no. 2, pp. 234-240, 2010

[6] P. Papadimitratos, A. de La Fortelle, K. Evensen, R. Brignolo, and S. Cosenza, "Vehicular communication systems: Enabling technologies, applications, and future outlook on intelligent transportation, IEEE Commun. Mag., vol. 11, no. 1, pp. 84-95, Nov. 2009.

[7] F. da Costa, "Rethinking the Internet of Things: A Scalable Approach to Connecting Everything, Apress, 1st Ed. 2014.

[8] B. C. Villaverde et at., "Service Discovery Protocols for Constrained Machine-to-Machine Communications", IEEE Commun. Surveys Tuts., vol. 16, no. 1, pp. 41-60, 2014.

[9] G. Wunder, M. Kasparick, and P. Jung., "Spline Waveforms and Interference Analysis for 5G Random Access with Short Message Support", Online. [Available]: http://arxiv.org/pdf/1501.02917.pdf

[10] G. Wunder, P. Jung, and C. Wang, "Compressive Random Access for Post-LTE Systems", In Proc. of the IEEE ICC - Workshop on Massive Uncoordinated Access Protocols, pp. 539-544, June 2014.

[11] S.-Y. Lien, K.-C. Chen, and Y. Lin, "Toward Ubiquitous Massive Accesses in 3GPP Machine-to-machine Communications", IEEE Commun. Mag., vol. 49, no. 4, pp. 66-74, Apr. 2011.

[12] M.-Y. Cheng, G.-Y. Lin, H.-Y. Wei, and A.-C. Hsu, "Overload control for machine-type-communications in LTE-advanced system", IEEE Commun. Mag., vol. 50, no. 6, pp. 38-45, Jun. 2012.

[13] M. Gerasimenko et al., "Energy and delay analysis of LTE-advanced RACH performance under MTC overload", In Proc. of the IEEE Globecom Workshops, pp. 1632-1637, Dec. 2012.

[14] U. Phuyal, A. Koc, M.-H. Fong, and R. Vannithamby, "Controlling access overload and signaling congestion in M2M networks", In Proc. of the Asilomar Conference on Signals, Systems, and Computers, pp. 591-595, Nov. 2012.

[15] T. L. Marzetta, "Noncooperative cellular wireless with unlimited numbers of base station antennas", IEEE Trans. Wireless Commun., vol. 9, no. 11, pp. 3590-3600, Nov. 2010.

[16] H. Q. Ngo, E. G. Larsson, and T. L. Marzetta, "Energy and spectral efficiency of very large multiuser MIMO systems, IEEE Trans. Commun., vol. 61, no. 4, pp. 1436-1449, Apr. 2013.

[17] Y. Shen and E. Martinez, "Channel Estimation in OFDM Systems, Freescale Semiconductor Application Note AN3059, Rev. 0, Jan. 2006.

[18] E. J. Candes, J. Romberg, and T. Tao, "Robust uncertainty principles: Exact signal reconstruction from highly incomplete frequency information, IEEE Trans. Inform. Theory, vol. 52, no. 2, pp. 489-509, Feb. 2006.
[19] D. Donoho, "Compressed sensing, IEEE Trans. Inform. Theory, vol. 52, no. 4, pp. 1289-1306, Apr. 2006.

[20] W. U. Bajwa, J. Haupt, A. M. Sayeed, and R. Nowak, "Compressed channel sensing: A new approach to estimating sparse multipath channels, Proc. IEEE, vol. 98, no. 6, pp. 1058-1076, Jun. 2010.

[21] C. R. Berger, Z. Wang, J. Huang, and S. Zhou, "Application of compressive sensing to sparse channel estimation, IEEE Commmun. Mag., vol. 48, no. 11, pp. 164-174, Nov. 2010.

[22] J. R. Barry, E. A. Lee, and D. G. Messerschmitt, "Digital Communication, Springer, 3rd Ed. 2004.

[23] F. Rusek et al., "Scaling up MIMO: Opportunities and Challenges with Very Large Arrays, IEEE Signal Process. Mag., vol. 30, no. 1, pp. 40-60, Jan. 2013.

[24] S. Verdu, "Multiuser Detection, Cambridge University Press, 1st Ed. 1998.

[25] Y. -C. Liang, E. Y. Cheu, L. Bai, and G. Pan, "On the relationship between MMSE-SIC and BI-GDFE receivers for large multiple-input multiple-output channels, IEEE Trans. Signal Process., vol. 56, no. 8, pp. 3627-3637, Aug. 2008.

[26] Felipe A. P. de Figueiredo, Fabbryccio A. C. M. Cardoso, Renato R. Lopes, and Joao Paulo Miranda, On the Application of Massive $M U-M I M O$ in the Uplink of Machine Type Communication Systems, International Workshop on Telecommunications (IWT), June 2015.

[27] Joao P. Miranda, Arman Farhang, Nicola Marchetti, Felipe A. P. de Figueiredo, Fabbryccio A. C. M. Cardoso, and FabrÃcio Figueiredo, On massive MIMO and its applications to machine type communications and FBMC-based networks, EAI Endorsed Transactions on Ubiquitous Environments, vol. 2, no. 5, July 2015.

[28] Felipe A. P. de Figueiredo, Fabiano Mathilde, Fabricio Santos, Fabbryccio A. C. M. Cardoso, and Gustavo Fraidenraich, On channel estimation for massive MIMO with pilot contamination and multipath fading channels, IEEE Latin American Conference on Communications (LATINCOM), October 2016.

[29] Felipe A. P. de Figueiredo, Fabbryccio A. C. M. Cardoso, Ingrid Moerman, and Gustavo Fraidenraich, Channel Estimation for Massive MIMO TDD Systems Assuming Pilot Contamination and Frequency Selective Fading, IEEE Access, vol. 5, no. 9, pp. 17733-17741, September 2017.

[30] Felipe A. P. de Figueiredo, Fabbryccio A. C. M. Cardoso, Ingrid Moerman, and Gustavo Fraidenraich, Channel estimation for massive MIMO TDD systems assuming pilot contamination and flat fading, EURASIP Journal on Wireless Communications and Networking, vol. 2018, no. 14, January, 2018.

[31] Felipe A. P. de Figueiredo, Fabbryccio A. C. M. Cardoso, Ingrid Moerman, Gustavo Fraidenraich, On the Application of Massive MIMO Systems to Machine Type Communications, IEEE Access, vol. 7, no. 12, pp. 2589-2611, December 2018. 